\title{
Latent class evaluation of a milk test, a urine test, and the fat-to-protein percentage ratio in milk to diagnose ketosis in dairy cows
}

\author{
M. A. Krogh, ${ }^{1}$ N. Toft, and C. Enevoldsen \\ Department of Large Animal Sciences, Faculty of Life Sciences, University of Copenhagen, Grønnegårdsvej 2, \\ DK-1870 Frederiksberg C, Denmark
}

\begin{abstract}
In this study, 3 commonly used tests to diagnose ketosis were evaluated with a latent class model to avoid the assumption of an available perfect test. The 3 tests were the KetoLac BHB (Sanwa Kagaku Kenkyusho Co. Ltd., Nagoya, Japan) test strip that tests milk for $\beta$-hydroxybutyrate, the KetoStix (Bayer Diagnostics Europe Ltd., Dublin, Ireland) test strip that tests urine for acetoacetate, and the fat-to-protein percentage ratio (FPR) in milk. A total of 8,902 cows were included in the analysis. The cows were considered to be a random sample from the population of Danish dairy cattle under intensive management, thus representing a natural spectrum of ketosis as a disease. All cows had a recorded FPR between 7 and $21 \mathrm{~d}$ postpartum. The KetoLac BHB recordings were available from 2,257 cows and 6,645 cows had a KetoStix recording. The recordings were analyzed with a modified Hui-Walter model, in a Bayesian framework. The specificity of the KetoLac BHB test and the KetoStix test were both high [0.99 (0.97-0.99)], whereas the specificity of FPR was somewhat lower $[0.79(0.77-0.81)]$. The best sensitivity was for the KetoStix test $[0.78(0.55-0.98)]$, followed by the FPR $[0.63(0.58-0.71)]$ and KetoLac BHB test [0.58 (0.35-0.93)].
\end{abstract}

Key words: ketosis, diagnostic test evaluation, latent class model, gold standard

\section{INTRODUCTION}

Ketosis is a common disease in adult cattle. It typically occurs in dairy cows in early lactation and is clinically characterized by nonspecific signs such as anorexia, milk loss, central nervous symptoms, and loss of body fat. The pathogenesis of ketosis involves a complex set of manifestations of excessive mobilization of body reserves of stored energy, mostly fat, due to a lack of balance between the cow's energy demand

Received September 13, 2010.

Accepted February 1, 2011.

${ }^{1}$ Corresponding author: mok@life.ku.dk for milk production and her energy intake. The cause of low energy intake can be related to poor quality of feedstuff, including ketogenic substances in the feed, other diseases like milk fever (post-parturient hypocalcaemia), or displaced abomasums. However, increased body condition score at calving may also in itself decrease feed intake (Broster and Broster, 1998).

A range of biochemical tests are available to diagnose ketosis. Measurements of BHBA in serum or milk and acetoacetate (AcAc) in urine probably are the most common. Several studies have used serum concentrations of BHBA of $1400 \mu \mathrm{mol} / \mathrm{L}$ as the suggested cut-off or even gold standard to define ketosis, but several cutoffs for the available tests have been suggested (Geishauser et al. 1998; Carrier et al., 2004; Iwersen et al. 2009). Previous studies on the accuracy (sensitivity and specificity) of available tests for ketosis that can be used in the herd (cow-side test) have primarily used serum levels of BHBA as the gold standard. However, BHBA and AcAc measurements vary for several reasons. For example, diurnal variation (Nielsen et al., 2003), high levels of somatic cells in the milk that will give falsepositive results of a BHBA test on milk (Jeppesen et al., 2006), temperature (Geishauser et al., 2000), time of reading of urine sticks that influences color changes (Jeppesen et al., 2006), and pure measurement error. Fat-to-protein percentage ratio (FPR) in the milk has also been suggested as an indication of ketosis (Duffield et al., 1997; Čejna and Chlàdek, 2005). Excessive mobilization of fat will be reflected in an elevation of fat percentage. Because protein percentage is rather stable and cow-specific, FPR should be an indicator of ketosis that is adjusted for a cow effect. Although neither of the above tests can be considered perfect, they are all relevant tools in the dairy herd from a practical point of view and evaluation of their performance under field conditions is needed. Also, neither level of BHBA in serum, nor any other tests are appropriate to define a gold standard to describe the dynamic level of metabolic stress the cow is exposed to. The term gold standard should only be applied to diagnostic tests that have a sensitivity and specificity of 1 . However, as an alternative, the term criterion standard is adopted 
by the American Medical Association and defined as a method having an established or widely accepted accuracy for determining a diagnosis, providing a standard to which a new screening or diagnostic test can be compared. Thus, previously obtained estimates of test accuracy for ketosis tests may be biased, due to the misclassification bias, which occurs from using a less than perfect test in a testing scheme to define cases and non-cases (Nielsen and Toft, 2002). Cow-side ketosis tests are ideally tools to detect ketosis at a stage where the possibly diffuse clinical symptoms are not yet present and not just to verify a clinical suspicion. This provides the practitioner the possibility to intervene efficiently. Thus, a practically relevant disease definition relates to cows in all stages of ketosis, rather than one subject to the selection bias imposed by a classification scheme using (e.g., serum as a gold standard or clinical symptoms).

Under certain conditions, latent class analysis (LCA) can be used to estimate the sensitivity (Se) and specificity (Sp) of diagnostic tests without the assumption of one being a gold standard (Toft et al., 2007a). The basic LCA relies on what is generally referred to as the Hui-Walter paradigm (Hui and Walter, 1980): 2 or more tests must be evaluated in 2 or more subpopulations with different prevalence of the disease, the tests must have constant Se and Sp across the populations, and the tests must be conditionally independent, given disease status. The LCA methods have gained increased acceptance as a means of evaluating diagnostic tests for infectious diseases and are now endorsed by the OIE (World Organization for Animal Health) as an alternative to classic test evaluations in the OIE fitness for purpose concept (OIE, 2010).

The objective of this study was to compare the test performance of a milk-based cow-side BHBA test, a urine-based cow-side AcAc test, and the FPR in milk as tools to diagnose ketosis without the assumption of an available gold standard.

\section{MATERIALS AND METHODS}

\section{Diagnostic Tests}

The KetoLac BHB (Sanwa Kagaku Kenkyusho Co. Ltd., Nagoya, Japan; marketed as KetoTest in the United States) test is a dip-stick that measures the milk contents of BHBA on a semiquantitative scale. In this study we used the cut-off $\geq 200 \mu \mathrm{mol} / \mathrm{L}$ of BHBA in milk, recommended by the manufacturer, to define a positive test. Tests were performed as described by the manufacturer. However, variation in the procedure between veterinarians might exist (such as preceding testing for mastitis).
The KetoStix (Bayer Diagnostics Europe Ltd., Dublin, Ireland) is a dip-stick that measures the AcAc content in the urine on a semiquantitative scale. A concentration of $4 \mathrm{mmol} / \mathrm{L}$ (moderate) or above of AcAc in urine was chosen as the cut-off for a positive KetoStix test (Carrier et al., 2004). Tests were performed as described by the manufacturer on urine collected as catheter sampling, spontaneous urination, or urination induced by manual manipulation the distal urethra in the vagina.

The FPR was calculated based on 1 of the 11 annual milk recordings from the Danish milk control program (RYK, 2010). Fat-to-protein percentage ratio values above 1.5 were defined as test positive for ketosis, as suggested by Čejna and Chlàdek (2005).

\section{Data Collection}

Data were extracted from the Veterinary Production and Consultancy (VPR) database, which is a subset of the National Danish Cattle Database (Krogh and Enevoldsen, 2006), on June 7, 2009. The VPR database consists of herds, where the veterinarians perform systematic clinical recordings on well-defined groups of cows. At least $12 \%$ of the Danish dairy herds have their cows routinely tested in early lactation for ketosis by a veterinarian. The veterinarians voluntarily submit cow-level registrations to the VPR database. In most Danish dairy herds, milk yield, fat percentage, and protein percentage are measured at up to 11 annual test days. The primary inclusion criteria for our study were that cows had been tested for ketosis with either the KetoLac BHB or the KetoStix between 7 and 21 $\mathrm{d}$ postpartum and had a milk test-date recording $1 \mathrm{~d}$ before the day of the ketosis test. The restriction with a minimum of $7 \mathrm{~d}$ postpartum was imposed because some cows might still have some colostrum in the milk up to this time point and colostrum will affect the measurement of fat content in the milk. Milk recordings at or after the day of testing for ketone bodies were also excluded because test results might have induced treatments of ketosis (usually glucocorticoids parentally or propylene glycol given orally; Radostits et al., 2000), which might affect FPR. Initial analyses of the data suggested that treatments could affect the milk composition very quickly. Records of ketosis treatment were available but not used, except for the initial data analyses. Out of 141,133 ketosis tests available from individual cows, a total of 8,902 cows were selected based on the milk test date. Of these, 2,257 cows were examined with the KetoLac BHB test and 6,645 cows with the KetoStix test. The KetoLac BHB tests were recorded from February 2004 to April 2009. The KetoStix tests were recorded between February 
2003 and April 2009. Both tests were recorded during all seasons of the year.

\section{Statistical Model}

To estimate the Se and Sp of the KetoStix, KetoLac $\mathrm{BHB}$, and FPR in the absence of a gold standard, we applied LCA, using a modified version of the Hui-Walter model (Hui and Walter, 1980). As previously mentioned, the Hui-Walter model assumes that prevalences are different across subpopulations, Se and Sp are constant (within tests) across the subpopulations, and tests are conditionally independent, given disease status. These assumptions imply that for each subpopulation a $2 \times$ 2 table of tabulated paired test results is required. For each combination of test results, the probability of that particular result can then be expressed in terms of Se, $\mathrm{Sp}$, and prevalence (p). For the $i$ th subpopulation, the joint probability $(\mathrm{Pr})$ of test $1\left(\mathrm{~T}_{1}\right)$ positive and test $2\left(\mathrm{~T}_{2}\right)$ negative can be expressed as $\operatorname{Pr}\left(\mathrm{T}_{1}+, \mathrm{T}_{2}-\right)=$ $\operatorname{Pr}\left(\mathrm{T}_{1}+, \mathrm{T}_{2}-\mid \mathrm{D}+\right) \operatorname{Pr}(\mathrm{D}+)+\operatorname{Pr}\left(\mathrm{T}_{1}+, \mathrm{T}_{2}-\mid \mathrm{D}-\right) \operatorname{Pr}(\mathrm{D}-)$ $=\operatorname{Pr}\left(\mathrm{T}_{1}+\mid \mathrm{D}+\right) \operatorname{Pr}\left(\mathrm{T}_{2}-\mid \mathrm{D}+\right) \operatorname{Pr}(\mathrm{D}+)+\operatorname{Pr}\left(\mathrm{T}_{1}+\mid \mathrm{D}-\right)$ $\operatorname{Pr}\left(\mathrm{T}_{2}-\mid \mathrm{D}-\right) \operatorname{Pr}(\mathrm{D}-)=\mathrm{Se}_{1}\left(1-\mathrm{Se}_{2}\right) \mathrm{p}_{\mathrm{i}}+\left(1-\mathrm{Sp}_{1}\right) \mathrm{Sp}_{2}(1$ - $\left.\mathrm{p}_{\mathrm{i}}\right)$, where, for example, $\operatorname{Pr}\left(\mathrm{T}_{1}+, \mathrm{T}_{2}-\mid \mathrm{D}-\right)$ means the joint probability of test 1 being positive $\left(\mathrm{T}_{1}+\right)$ and test 2 being negative $\left(\mathrm{T}_{2}-\right)$, conditional on $(\mid)$, the test subject being truly disease free $(\mathrm{D}-)$. The first equation takes into account that, although unobserved, the true state of each animal is either diseased or not diseased. The second equation uses the assumption of conditional independence, given disease status, to express the joint probability as a product. The final equation merely rewrites the expression using the conventional terms of $\mathrm{Se}, \mathrm{Sp}$, and $\mathrm{p}$. The above expression uses 5 terms $\left(\mathrm{Se}_{1}\right.$, $\mathrm{Sp}_{1}, \mathrm{Se}_{2}, \mathrm{Sp}_{2}$, and $\mathrm{p}_{\mathrm{i}}$ ); the other 3 probabilities in the $2 \times 2$ table may be expressed similarly using the same 5 terms. Thus, each $2 \times 2$ table of data representing a subpopulation can be expressed using 5 parameters, while providing only 3 degrees of freedom in the data. Hence, identifiability of the original 2-test, 2-population Hui-Walter model was given by the assumptions of conditional independence, given disease status; constant test properties across populations; and the prevalences truly differing between populations. These assumptions ensured that for 2 populations, 2 times 3 (i.e., 6 degrees of freedom) were available and only 6 parameters were used, because adding a population only meant adding 1 new parameter (the prevalence of the population). We shall address the validity of these assumptions for our model further in the discussion.

The original Hui-Walter 2-test, 2-population model was extended as illustrated in Figure 1. Thus, the Se and Sp of the KetoLac BHB test were estimated from subpopulation 1, 2, and 3; the Se and Sp of the KetoStix from subpopulation 4, 5, and 6; and the Se and Sp of the FPR were estimated using all 6 subpopulations. The 6 subpopulations were defined as follows: subpopulation 1 and 4 are first-parity cows, subpopulation 2 and 5 are second-parity cows, and subpopulation 3 and 6 are older than second parity. Essentially, we have a 2-test, 3-population model for each BHBA and FPR test combination, giving us 9 degrees of freedom, while only requiring 7 parameters. Furthermore, because the FPR is assumed to be constant across all populations, the full model uses only 12 parameters with 18 degrees of freedom.

We chose a Bayesian estimation approach (Branscum et al., 2005) implemented in OpenBUGS software (Thomas et al., 2006), which uses a Markov Chain Monte Carlo (MCMC) sampling algorithm to obtain a Monte Carlo (MC) sample from the posterior distribution. The first 10,000 MC samples were discarded as a burn-in to allow convergence, and the following 90,000 iterations were used for posterior inference. Convergence of the MCMC chain after the initial burn-in was assessed by visual inspection of the time-series plots of selected variables as well as Gelman-Rubin diagnostic plots using 3 sample chains with different initial values (Toft et al., 2007b).

In Bayesian analysis, all parameters are modeled using distributions, where prior distributions are provided to reflect what is known about the tests. However, as we used a model capable of estimating all parameters from data alone, we chose priors in the shape of uniform distributions on the interval between 0 and 1, modeled using the Beta $(1,1)$ distribution for all parameters (i.e., Se and Sp of the 3 tests and prevalence of the 6 populations). This implies that posterior estimates are comparable to those obtained from a maximum likelihood analysis. However, we avoided the questionable assumptions about asymptotic normality of the estimates (Toft et al., 2005).

Based upon the estimates of Se and Sp of the 3 tests, we calculated the combined Se and Sp of FPR and the KetoLac BHB/KetoStix tests. An assumption for this calculation is the conditional independence of the tests (Gardner et al. 2000). Due to this issue the combined test performance of the KetoLac BHB and KetoStix test is not calculated. Both a parallel and a serial testing scheme were demonstrated. Parallel testing means that the combined test is only considered negative if, and only if, both tests are negative. The parallel diagnostic Se $\left(S e_{\mathrm{par}}\right)$ and parallel diagnostic Sp $\left(S p_{\mathrm{par}}\right)$ are calculated as $S e_{\mathrm{par}}=1-\left(1-S e_{1}\right)\left(1-S e_{2}\right)$ and $S p_{\mathrm{par}}=$ $S p_{1} S p_{2}$, where $S e_{1}$ and $S p_{1}$ are the diagnostic properties of test 1 and $S e_{2}$ and $S p_{2}$ are the diagnostic proper- 


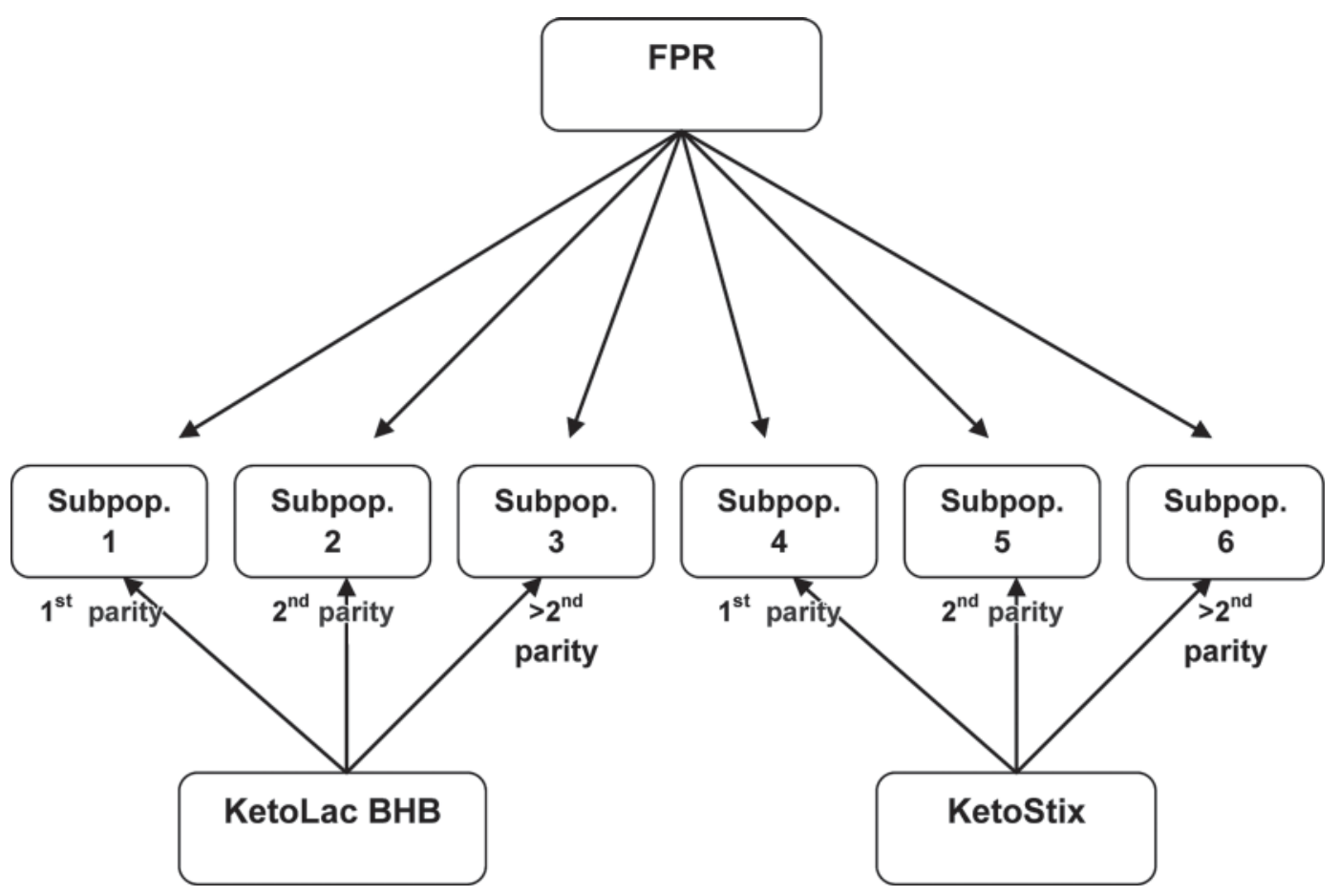

Figure 1. Study design that reflects the applied Bayesian latent class model. The data are divided into population 1, 2, and 3 versus 4,5 , and 6 based on type of cow-side ketosis test [KetoLac BHB (Sanwa Kagaku Kenkyusho Co. Ltd., Nagoya, Japan) or KetoStix (Bayer Diagnostics Europe Ltd., Dublin, Ireland)]. For each type of ketosis test, these recordings are divided into parity groups. Fat-to-protein percentage ratio $(\mathrm{FPR})$ is measured on all of the cows. Subpop. = subpopulation.

ties of test 2. With parallel testing, the Se will often be higher than for any of the tests alone, whereas the Sp often will be substantially lower. In a serial testing scheme, both tests need to be positive for the combined test to be positive. The serial diagnostic Se $\left(S e_{\text {ser }}\right)$ and parallel diagnostic $\operatorname{Sp}\left(S p_{\text {ser }}\right)$ are calculated as $S e_{\text {ser }}=$ $S e_{1} S e_{2}$ and $S p_{\text {ser }}=1-\left(1-S p_{1}\right)\left(1-S p_{2}\right)$, where $S e_{1}$ and $S p_{1}$ are the diagnostic properties of test 1 and $S e_{2}$ and $S p_{2}$ are the diagnostic properties of test 2 .

Posterior inference was based on medians and 95\% posterior credibility intervals (PCI, the Bayesian analog of a confidence interval) of the prevalence in the 6 subpopulations, the Se and Sp of the 3 tests, and the combined Se and Sp of the tests.

\section{RESULTS}

To satisfy the modeling assumptions, data were divided into the subpopulations defined by Figure 1 . Summary statistics of the number of cows, the number of herds, and the median number of cows from each herd, as well as the percentage of breeds in each population, are given in Table 1.

It is apparent that most herds only contributed with few cows to the analysis. The distribution of breeds in-

Table 1. Summary statistics of the sample population: number of cows, number of herds, median number of cows within herds, and breed distribution in parity groups within type of ketone body test $\left(\right.$ KetoLac BHB ${ }^{1}$ or KetoStix ${ }^{2}$ )

\begin{tabular}{|c|c|c|c|c|c|c|}
\hline Item & \multicolumn{3}{|c|}{ KetoLac BHB } & \multicolumn{3}{|c|}{ KetoStix } \\
\hline Herds (n) & 140 & 127 & 150 & 254 & 248 & 255 \\
\hline Cows with ketone tests in herd (median $\mathrm{n}$ ) & 3 & 3 & 3 & 6 & 5 & 5 \\
\hline Red Danish cattle (\%) & 14 & 12 & 11 & 8 & 7 & 8 \\
\hline
\end{tabular}

${ }^{1}$ Sanwa Kagaku Kenkyusho Co. Ltd., Nagoya, Japan.

${ }^{2}$ Bayer Diagnostics Europe Ltd., Dublin, Ireland. 
Table 2. Cross-tabulation of the dichotomized test results for fat-to-protein percentage ratio (FPR; cut-off: $>1.5$ ), KetoLac BHB ${ }^{1}$ (cut-off: $\geq 200 \mu \mathrm{mol} / \mathrm{L}$ ), and KetoStix ${ }^{2}$ (cut-off: $\geq 4 \mathrm{mmol} / \mathrm{L}$ ), stratified by the use of either KetoLac BHB or KetoStix and, subsequently, into parity groups

\begin{tabular}{|c|c|c|c|}
\hline Group & Ketone test & $\mathrm{FPR}+$ & FPR - \\
\hline \multicolumn{4}{|l|}{ KetoLac BHB } \\
\hline \multirow[t]{2}{*}{ Subpopulation 1 (first parity) } & KetoLac BHB + & 21 & 24 \\
\hline & KetoLac BHB - & 202 & 548 \\
\hline \multirow{2}{*}{ Subpopulation 2 (second parity) } & KetoLac BHB + & 19 & 9 \\
\hline & KetoLac BHB - & 100 & 492 \\
\hline \multirow[t]{2}{*}{ Subpopulation 3 (>second parity) } & KetoLac BHB + & 46 & 39 \\
\hline & KetoLac BHB - & 176 & 581 \\
\hline \multicolumn{4}{|l|}{ KetoStix } \\
\hline \multirow[t]{2}{*}{ Subpopulation 4 (first parity) } & KetoStix + & 155 & 64 \\
\hline & KetoStix - & 600 & 1,733 \\
\hline \multirow[t]{2}{*}{ Subpopulation 5 (second parity) } & KetoStix + & 67 & 72 \\
\hline & KetoStix - & 357 & 1,399 \\
\hline \multirow[t]{2}{*}{ Subpopulation 6 (>second parity) } & KetoStix + & 197 & 139 \\
\hline & KetoStix - & 405 & 1,457 \\
\hline
\end{tabular}

${ }^{1}$ Sanwa Kagaku Kenkyusho Co. Ltd., Nagoya, Japan.

${ }^{2}$ Bayer Diagnostics Europe Ltd., Dublin, Ireland.

dicates that the KetoStix test is more frequently used to test Danish Holstein cows than the KetoLac BHB test.

The cross-tabulation of the test results dichotomized by the selected cut-offs and stratified by ketone test and parity group are given in Table 2. Based on the data in Table 2, the Se and Sp of the tests and the prevalences in the 6 subpopulations were estimated (Table 3). Slight differences in the parity-specific prevalences of ketosis were present for the populations defined by their use of KetoLac BHB or KetoStix. The overall prevalences of ketosis in the populations defined by the use of KetoLac BHB or KetoStix were 0.10 and 0.12 , respectively.

Table 4 shows the results from the combination of tests. Applying the KetoLac BHB test and the FPR test or the KetoStix and FPR in parallel substantially increased the Se, whereas only a minor decrease occurred in the Sp. A serial testing scheme will decrease the Se of the combined test substantially, whereas the Sp here was around 1.

\section{DISCUSSION}

The LCA creates a probabilistic disease definition based on the cross-classified test results. Given the tests applied in this study, the definition of ketosis in this study will be a condition between 7 and $21 \mathrm{~d}$ postpartum that manifests itself with either elevated levels of ketone bodies in milk or urine, or elevated fat content in milk. These indicators are all very likely to be manifestations of excessive mobilization of fat.

Table 3. Medians and 95\% posterior credibility intervals (PCI) from the latent class model using data from Table 2

\begin{tabular}{|c|c|c|}
\hline Parameter $^{1}$ & Median & $95 \%$ PCI \\
\hline \multicolumn{3}{|l|}{ KetoLac BHB } \\
\hline First parity, prevalence & 0.08 & $0.04-0.17$ \\
\hline Second parity, prevalence & 0.06 & $0.03-0.10$ \\
\hline$>$ Second parity, prevalence & 0.15 & $0.08-0.23$ \\
\hline \multicolumn{3}{|l|}{ KetoStix } \\
\hline First parity, prevalence & 0.10 & $0.07-0.16$ \\
\hline Second parity, prevalence & 0.08 & $0.05-0.12$ \\
\hline$>$ Second parity, prevalence & 0.18 & $0.14-0.26$ \\
\hline $\mathrm{Se}_{\text {KetoLac BHB }}{ }^{2}$ & 0.58 & $0.35-0.93$ \\
\hline Sp $_{\text {KetoLac BHB }}{ }^{2}$ & 0.99 & $0.97-0.999$ \\
\hline $\mathrm{Se}_{\mathrm{FPR}}{ }_{3}$ & 0.63 & $0.58-0.71$ \\
\hline $\mathrm{Sp}_{\mathrm{FPR}}{ }^{3}$ & 0.79 & $0.77-0.81$ \\
\hline $\mathrm{Se}_{\mathrm{KetoStix}}{ }_{4}$ & 0.78 & $0.55-0.98$ \\
\hline $\mathrm{Sp}_{\text {KetoStix }}$ & 0.99 & $0.98-0.999$ \\
\hline
\end{tabular}


Table 4. Medians and 95\% posterior credibility intervals (PCI) of the combined test performance of KetoLac BHB and KetoStix with fat-to-protein percentage ratio (FPR) in serial (ser) and parallel (par) ${ }^{1}$

\begin{tabular}{lcccc}
\hline Item & $\mathrm{Se}_{\text {par }}$ & $\mathrm{Sp}_{\text {par }}$ & $\mathrm{Se}_{\text {ser }}$ & $\mathrm{Sp}_{\text {ser }}$ \\
\hline KetoLac BHB-FPR & $0.85(0.75-0.98)$ & $0.77(0.75-0.80)$ & $0.37(0.22-0.60)$ & $0.99(0.99-1.00)$ \\
KetoStix-FPR & $0.92(0.83-0.99)$ & $0.78(0.76-0.81)$ & $0.49(0.34-0.64)$ & $0.99(0.99-1.00)$ \\
\hline
\end{tabular}

${ }^{1}$ KetoLac BHB (Sanwa Kagaku Kenkyusho Co. Ltd., Nagoya, Japan); KetoStix (Bayer Diagnostics Europe Ltd., Dublin, Ireland); Se = sensitivity; Sp = specificity.

We used LCA to estimate the sensitivity and specificity of KetoLac BHB, KetoStix, and FPR as tests for ketosis, without the assumption of an available gold standard test. The analysis showed that no real differences existed in median estimates of the $\mathrm{Sp}$ of the KetoLac BHB and KetoStix, but both were more specific than FPR. The KetoStix had the best median Se, followed by FPR, and KetoLac BHB, with noticeable differences in median estimates between all 3 tests. Combining KetoLac BHB and FPR or KetoStix and FPR in parallel substantially increased the Se of the test and decreased Sp, whereas the serial test combination substantially decreased Se and increased Sp.

One of the underlying assumptions in the LCA is a direct association between the (latent) disease state and the diagnostic tests included in the analysis. This means that it, to some extent, is the diagnostic tests under evaluation that define our disease. Our data did not allow us to estimate the relation between the latent disease definition derived from this study and the commonly used criterion standard of serum BHBA concentration of 1,400 $\mu \mathrm{mol} / \mathrm{L}$ (Radostits et al., 2000). However, it is possible to compare the results of other diagnostic test evaluations to our findings. Using a criterion standard of 1,400 $\mathrm{mol} / \mathrm{L}$ in serum and a cut-off of $\geq 200 \mu \mathrm{mol} / \mathrm{L}$ for the KetoLac BHB test, Carrier et al. (2004) found $\mathrm{Se}=0.27$ and $\mathrm{Sp}=0.99$; Geishauser et al. (2000) found $\mathrm{Se}=0.59$ and $\mathrm{Sp}=0.90$; and Iwersen et al. (2009) found $\mathrm{Se}=0.30$ with a $95 \%$ CI $(0.07$; 0.65 ) and $\mathrm{Sp}=0.98$. Another study by Geishauser et al. (1998) with the same cut-off but with a criterion standard of $1,200 \mu \mathrm{mol}$ of BHBA/L in serum, found Se $=0.45$ and $\mathrm{Sp}=0.97$.

Carrier et al. (2004) also studied the KetoStix using serum contents of BHBA of $1,400 \mu \mathrm{mol} / \mathrm{L}$, as a case definition using our selected cut-off of $4 \mathrm{mmol} / \mathrm{L}$ and found $\mathrm{Se}=0.49$ and $\mathrm{Sp}=0.99$; Iwersen et al. (2009) found $\mathrm{Se}=0.67$ and $\mathrm{Sp}=1.00$.

Duffield et al. (1997) have published Se and Sp of the protein-to-fat ratio $(1 / \mathrm{FPR})$ based on a criterion standard of serum BHBA at 1,200 $\mu \mathrm{mol} / \mathrm{L}: \mathrm{Se}=0.22$ and $\mathrm{Sp}=0.85$. The rather low $\mathrm{Se}$ in this study could be due to the study population (entire lactation) and a rather long time span between the 2 tests.
Overall, the LCA results estimated in this study are similar to the ones based on selected cut-offs of BHBA serum levels. However, using LCA, the estimates of test accuracy are not subject to the misclassification bias imposed by using an imperfect test as a gold standard, nor will tests be punished by correctly identifying true positives or negatives, which are misclassified by the imperfect gold standard, and thereby underestimate or overestimate the Se and Sp of the new test. Even though the results we find with the LCA are similar to the ones found in gold standard studies, we do not suggest that the gold standard studies are not subject to misclassification bias. Our findings may only imply that the amount of misclassification bias most likely is not large enough to substantially change the final result. Also, most other studies have been performed in small/medium-scale experiments, which also tend to eliminate important covariates, thereby providing a better result of test performance than can be found under field conditions.

The data used in our study were collected from a large number of different herds, with different management levels, seasons, and years, different breeds, and distribution of parities. Essentially, we obtained a random sample because the cows were selected based on their milk recording data and calving dates. Thus, the cases in our data can be considered a random sample of naturally affected cows, representing ketosis as it is manifest in intensive dairy systems with intensive veterinary services. Data were collected over several years, which should remove possible systematic effects of years and seasons. Thus, the estimates for Se and Sp of the 3 tests obtained in this study reflect the values of these tests when applied under field conditions in the intensive Danish dairy population.

Our motivation for using the FPR was two-fold: FPR linked the KetoLac BHB and the KetoStix because no cows were tested using both, and FPR is freely available. Despite the FPR clearly being less accurate than the other cow-side tests due to a low Sp, it may still have value for establishing and monitoring the herd prevalence of ketosis. Because the milk recordings are already being performed, this prevalence could work as an effective and cheap monitoring tool for ketosis on 
herd level, given that the sampling scheme and the Se and Sp of FPR is taken into account. In the near future, in-herd analysis of fat and protein percentage may be available, thus increasing the interest in the FPR as a useful monitor for ketosis on individual cow level. Because the Sp of the FPR test is low (0.79), Se is 0.63, and the prevalence of ketosis is below $20 \%$, numerically more false positives than true positives will occur. Applying an additional test, such as KetoStix, in a parallel testing scheme will not improve this, but more of the true positives will be detected $(\mathrm{Se}=0.92)$. Using KetoStix in a serial testing scheme will improve the overall Sp but the overall Se will drop to 0.49. Application of a routine testing scheme based on FPR alone or in combination with other tests should take into account what the herd manager wants to achieve by using these tests, the cost related to testing, the negative effects of having a false-negative result (diseased but untreated), the cost of treating cows that are not diseased (false negatives), and possible positive or negative effects of treatment.

The overall median prevalences of ketosis in the 2 populations defined by the use of KetoLac BHB or KetoStix were 0.10 and 0.12 , respectively. Prevalence estimates from the parity groups tested with the KetoStix were all larger than for the parity groups tested with the KetoLac BHB. However, the same pattern of secondparity cows having the lowest prevalence, followed by first-parity cows, and with old cows having the highest prevalence was found for both sets of subpopulations. Other studies (Dohoo and Martin, 1984; Nielen et al., 1994) have found prevalence estimates of ketosis similar to what we have found here. The difference in overall prevalence may be attributed to sampling variation or systematic differences in measurement protocols. However, it may also indicate that measurements of AcAc in urine and BHBA in milk are associated with different stages of the disease process of ketosis.

It is a requirement of the LCA model assumptions that prevalences differ between subpopulations. The effects on model performance of differences in prevalences in the subpopulations have been studied by Toft et al. (2005) on simulated data. The overall conclusions were that if the differences in prevalences in the subpopulations are small $(<10 \%)$, then the uncertainty and bias related to the estimates of sensitivity and specificity increase for a given sample size. However, considering the true overall prevalence in our study, it would be difficult to increase the differences between populations while ensuring the other assumptions of the Hui-Walter model. Furthermore, in our model, we use essentially 3 populations for each of the BHBA tests and 6 for the FPR.
We chose to split into subpopulations based on parity. Although this is generally not recommended for infectious diseases, where it most likely will cause differences in test properties, it is not considered a problem for this analysis. The BHBA contents in milk or the urine contents of AcAc will reflect the underlying pathophysiological process within the cow. It is unlikely that this pathophysiological process should be different for different parity groups. It is well known that the milk production increases with increasing parity group. However, there is no reason why (or evidence suggesting) the ratio between fat and protein in milk should be different for different parities and, hence, provide a different substrate for the growth of the calf, which must be considered the basic biological objective of milk production.

The concept of conditional independence between tests, given disease status, can best be understood by considering the opposite: conditional dependence between tests, given disease status. This often occurs when tests are based on the same phenomenon (e.g., 2 ELISA tests to detect antibodies): assuming that the true disease status is known (e.g., the test subject is truly infected), then knowing that test 1 is positive (or negative) will most likely influence the belief in a positive test result for test 2 , because now additional information considering the level of antibodies or possible cross-reactions can be incorporated in the belief of a test result of test 2, when applied to the known truly infected animal. From a biological point of view, it is hard to imagine that the KetoStix test for AcAc in urine and the FPR in milk should be conditionally dependent, given disease status, because they measure different substances in different body fluids from the cow. The conditional dependence or independence of the KetoLac BHB test for BHBA in milk and the KetoStix test for AcAc in urine is irrelevant because no cows were tested with both tests. It could be hypothesized that the excretion of BHBA in milk is linked to the fat molecules in the milk, so that higher fat percentage in the milk will give higher concentrations of BHBA in milk. This, however, is not possible to test using the available data.

\section{CONCLUSIONS}

Using LCA, we were able to estimate the Se and Sp of 3 tests for ketosis without the assumption of a gold standard. This enabled us to use a large data set with a random sample of naturally occurring stages of ketosis similar to what must be expected in the population and context where the tests are to be used. Thereby, we avoided the misclassification bias often seen in tra- 
ditional test evaluations due to the use of an imperfect test or a selection scheme to establish cases and controls. Our findings were that KetoStix had the highest sensitivity and specificity: 0.78 and 0.99 , respectively. The KetoLac BHB test had a similarly high specificity (0.99) but the lowest sensitivity (0.58). The FPR had a marginally higher sensitivity (0.63) than the KetoLac $\mathrm{BHB}$ and a substantially lower specificity (0.78).

\section{REFERENCES}

Branscum, A. J., I. A. Gardner, and W. O. Johnson. 2005. Estimation of diagnostic-test sensitivity and specificity through Bayesian modeling. Prev. Vet. Med. 68:145-163.

Broster, W. H., and V. J. Broster. 1998. Body score of dairy cows. J. Dairy Res. 65:155-173.

Carrier, J., S. Stewart, S. Godden, J. Fetrow, and P. Rapnicki. 2004. Evaluation and use of three cowside tests for detection of subclinical ketosis in early postpartum cows. J. Dairy Sci. 87:37253735 .

Čejna, V., and G. Chládek. 2005. The importance of monitoring changes in milk fat to milk protein ratio in Holstein cows during lactation. J. Cent. Eur. Agric. 6:539-546.

Dohoo, I. R., and S. W. Martin. 1984. Subclinical ketosis: Prevalence and associations with production and disease. Can. J. Comp. Med. 48:1-5.

Duffield, T. F., D. F. Kelton, K. E. Leslie, K. D. Lissemore, and J. H. Lumsden. 1997. Use of test day milk fat and milk protein to detect subclinical ketosis in dairy cattle in Ontario. Can. Vet. J. 38:713-718.

Gardner, I. A., H. Stryhn, P. Lind, and M. T. Collins. 2000. Conditional dependence between tests affects the diagnosis and surveillance of animal diseases. Prev. Vet. Med. 45:107-122.

Geishauser, T., K. Leslie, D. Kelton, and T. Duffield. 1998. Evaluation of five cowside tests for use with milk to detect subclinical ketosis in dairy cows. J. Dairy Sci. 81:438-443.

Geishauser, T., K. Leslie, J. Tenhag, and A. Bashiri. 2000. Evaluation of eight cow-side ketone tests in milk for detection of subclinical ketosis in dairy cows. J. Dairy Sci. 83:296-299.

Hui, S. L., and S. D. Walter. 1980. Estimating the error rates of diagnostic tests. Biometrics 36:167-171.

Iwersen, M., U. Falkenberg, R. Voigtsberger, D. Forderung, and W. Heuwieser. 2009. Evaluation of an electronic cowside test to detect subclinical ketosis in dairy cows. J. Dairy Sci. 92:2618-2624. doi:10.3168/jds.2008-1795.

Jeppesen, R., J. M. D. Enemark, and C. Enevoldsen. 2006. Ketone body measurement in dairy cows. Reference OS43-2 in Proc. 24th
World Buiatrics Congress, Nice, France. World Assoc. Buiatrics, Vienna, Austria.

Krogh, M. A., and C. Enevoldsen. 2006. Organizational and educational support to dairy herd health programs. Page 133 in Proc. Int. Soc. Vet. Epidemiol. Econ., Queensland, Australia. Accessed Dec. 12, 2008. http://www.sciquest.org.nz/crusher_download. asp? article $=10003577$.

Nielen, M., M. G. A. Aarts, A. G. M. Jonkers, T. Wensing, and Y. H. Schukken. 1994. Evaluation of two cowside tests for the detection of subclinical ketosis in dairy cows. Can. Vet. J. 35:229-232.

Nielsen, N. I., K. L. Ingvartsen, and T. Larsen. 2003. Diurnal variation and the effect of feed restriction on plasma and milk metabolites in TMR-fed dairy cows. J. Vet. Med. A Physiol. Pathol. Clin. Med. 50:88-97.

Nielsen, S. S., and N. Toft. 2002. Optimisation of the validity of ELISA and faecal culture tests for paratuberculosis: Selection of population or correction by population characteristics? Pages 400-405 in Proc. 7th Int. Colloq. Paratuberculosis, June 11-14, 2002, Bilbao, Spain. International Association for Paratuberculosis, Inc., Madison, WI.

OIE. 2010. Principles and methods of validation of diagnostic assays for infectious diseases. Chapter 1.1.4/5. Pages 1-18 in OIE Terrestrial Manual 2010. Accessed March 8, 2011. http://www.oie.int/fileadmin/Home/eng/Health_Standards/tahm/1.1.04_VALID.pdf.

Radostits, O. M., C. C. Gay, D. C. Blood, and K. W. Hinchcliff. 2000 Ketosis in ruminants. Pages 1452-1456 in Veterinary Medicine: A Textbook of the Diseases of Cattle, Sheep, Pigs, Goats and Horses. 9th ed. O. M. Radostits, C. C. Gay, D. C. Blood, and K. W. Hinchcliff, ed. Elsevier Health Services. Harcourt Publishers Limited, London, UK.

RYK. 2010. Service Technology Health. RYK-Livestock Registration and Milk Recording. Agro Food Park, Udkaersvej 15, DK-8200 Aarhus N, Denmark. Accessed Sep. 11, 2010. http://www.landbrugsinfo.dk/Kvaeg/RYK/Sider/RYKfolder_english.mht.

Thomas, A., B. O'Hara, U. Ligges, and S. Sturtz. 2006. Making BUGS open. R News 6/1:12-17. ISSN 1609-3631. Accessed March 8, 2010. http://cran.r-project.org/doc/Rnews/Rnews_2006-1.pdf.

Toft, N., J. Åkerstedt, J. Tharaldsen, and P. Hopp. 2007a. Evaluation of three serological tests for diagnosis of Maedi-Visna virus infection using latent class analysis. Vet. Microbiol. 120:77-86.

Toft, N., G. T. Innocent, G. Gettinby, and S. W. J. Reid. 2007b. Assessing the convergence of Markov Chain Monte Carlo methods: An example from evaluation of diagnostic tests in absence of a gold standard. Prev. Vet. Med. 79:244-256.

Toft, N., E. Jørgensen, and S. Højsgaard. 2005. Diagnosing diagnostic tests: Evaluating the assumptions underlying the estimation of sensitivity and specificity in the absence of a gold standard. Prev. Vet. Med. 68:19-33. 